\title{
DISSOLUTION ENHANCEMENT OF TETRAHYDROCURCUMIN USING OPTIMIZED SELF-NANOEMULSIFYING DRUG DELIVERY SYSTEM
}

\author{
IKA YUNI ASTUTI*, TRI SULIATIN, RETNO WAHYUNINGRUM
}

Department of Pharmaceutical Technology, Faculty of Pharmacy, Universitas Muhammadiyah Purwokerto, Indonesia.

Email: ikayuniastuti@ump.ac.id

Received: 25 April 2019, Revised and Accepted: 19 July 2019

\section{ABSTRACT}

Objective: The objective of this study was to optimize and enhance the dissolution of tetrahydrocurcumin (THC) using optimized self-nanoemulsifying drug delivery system (SNEDDS).

Methods: The optimization was carried out with the D-optimal design using software design expert 7.15. The independent variables were the amount of oil, surfactant, and cosurfactant. The dependent variables were the emulsification time, percentage transmittance, and dissolution efficiency at minute 15 (DE15). The dissolution profile of the THC in optimum formulation compared with the unmodified THC.

Results: The optimum formulation of SNEDDS consists of 10\% Labrafil, 80\% Kolliphor:Labrasol (1:3), and 10\% PEG 400. The DE15 was 49.45\%, significantly higher than the THC unmodified $(\mathrm{p}<0.05)$. The estimated responses were not significantly different from the experimental responses. The droplet size of the optimum formula was $53.8 \mathrm{~nm}$, the polydispersity index was 0.043 and the zeta value was $-19.4 \mathrm{mV}$.

Conclusion: The D-optimal design was successfully determining the optimum formula of THC loaded SNEDDS. The SNEDDS formulation was increased the dissolution of THC.

Keywords: Dissolution, Optimization, Tetrahydrocurcumin, Self-nanoemulsifying drug delivery system, D-Optimal design.

(C) 2019 The Authors. Published by Innovare Academic Sciences Pvt Ltd. This is an open access article under the CC BY license (http://creativecommons. org/licenses/by/4. 0/) DOI: http://dx.doi.org/10.22159/ijap.2019.v11s5.T0079

\section{INTRODUCTION}

Tetrahydrocurcumin (THC) is an active metabolite of curcumin, a yellow dye found in turmeric (Curcuma longa $\mathrm{L}$.). Compared to the other curcuminoids, THC shows a stronger antioxidant and anti-inflammation activity [1]. The docking analysis showed that THC might be able to inhibit amyloid precursor protein (APP) so that it has the potential to be used in the treatment of Alzheimer's disease [2]. Although THC has potent activity, the bioavailability of THC is limited due to its poor water solubility and absorption. Several approaches to increase the solubility of THC have been carried out. These approaches include THC formulations into solid dispersions and inclusion complexes [3,4]. Both of these formulations succeeded in increasing the solubility of THC, but each had limitations. Solid dispersion causes the active substance to be in an amorphous state so that its solubility in water increases. However, crystallization can occur during the manufacturing process in the presence of mechanical stress or during storage due to temperature and humidity stresses, whereas inclusion complexes have limited drug loading.

A promising strategy to enhance drug solubility and absorption is a self-nanoemulsifying drug delivery system (SNEDDS). This system is an isotropic mixture of oil, surfactant, and cosurfactant. When dispersing the SNEDDS containing an hydrophobic drug to water, the drug in oil nanodroplet will quickly spread and emulsified, which causes an increase in the drug solubility, dissolution, and absorption. The SNEDDS formulation was reported to have succeeded in increasing the dissolution of rivaroxaban, curcumin, duloxetine hydrochloride, and pentagamavunon-0 [5-7].

The quality of SNEDDS is highly influenced by the composition of oil, surfactant, and cosurfactant. An effective and efficient method to optimize the composition and analyze the effect of the components is by an experimental design. D-optimal mixture design allows the addition of existing design for a more flexible model than the models offered by simplex lattice design [8]. Thus, the aims of this research are to optimize the SNEDDS formulation of THC using D-optimal design for dissolution enhancement of the THC.

\section{MATERIALS AND METHODS}

\section{Materials}

THC (technical grade, Xi'an Tianxingjian Natural Bio product Co. Ltd, Shaanxi, China), oleic acid, PEG 400, span 80, Tween 80, and Tween 20 (technical grade, Sigma-Aldrich). Labrafil, Labrasol, and Transcutol technical grade was kindly provided by Gattefosse, France. Kolliphor and Myritol (technical grade, PT BASF Indonesia) and methanol (spectrophotometric grade, Merck) were used.

\section{METHODS}

\section{Preliminary studies}

Five hundred milligrams of THC were accurately weighed, added to $1 \mathrm{ml}$ of a vehicle in a microtube, vortexed for $5 \mathrm{~min}$ and stirred for $24 \mathrm{~h}$. After stirring, the microtube was incubated at room temperature for $24 \mathrm{~h}$, and then centrifuged at $6000 \mathrm{rpm}$ for $10 \mathrm{~min}$. The supernatant was diluted in methanol, and the soluble THC in the vehicle was determined by Ultraviolet (UV)-visible spectrophotometric method. The best oil, surfactant, and cosurfactant in dissolving THC were selected for further study.

The compatibility between SNEDDS components performed by vortexing the mixture of selected surfactant Kolliphor:Labrasol (1:3), co-surfactant (PEG400), and PEG400 at a various ratio of $10 \%-90 \%$ of each component. After allowed for $24 \mathrm{~h}$, the mixture was observed for any separation.

\section{Construction of the pseudoternary phase diagram}

The mixtures from the compatibility study were studied for the selfnanoemulsifying properties. A $100 \mu \mathrm{l}$ of the mixture were introduced 
to $100 \mathrm{ml}$ of water under magnetic stirring at $50 \mathrm{rpm}$. The time interval from the introducing to changes in the emulsion clarity was measured and noted as emulsification time. The percentage transmittance of the emulsion formed then was measured using visible spectrophotometry at $\lambda$ of $650 \mathrm{~nm}$. The emulsification time $\leq 2 \mathrm{~min}$ and percentage transmittance $\geq 80 \%$ without any phase separation was considered to have self-nanoemulsifying properties. The points of composition where the nanoemulsion formed spontaneously were be marked as a black triangle.

\section{Experimental design}

The experimental design for SNEDDS containing THC in this study used three factors (independent variables), i.e.,the oil, surfactant, and cosurfactant. Three responses (dependent variables) were tested, i.e., emulsification time, percentage transmittance, and dissolution efficiency at minute $15\left(\mathrm{DE}_{15}\right)$. The highest and lowest percentage of oil, surfactant, and cosurfactant which showed self-nanoemulsion properties was used as the high and low levels of the design constraint of D-optimal design.

\section{Preparation of SNEDDS containing THC}

The SNEDDS blanks were made by mixing the oil, surfactant, and cosurfactant based on the predetermined runs made by the DesignExpert 7.0 software. One milliliter of SNEDDS blanks was added with $97.45 \mathrm{mg}$ of THC, vortexed and sonicated until the THC completely dissolved. The SNEDDS were tested for the emulsification time, percentage transmittance, and $\mathrm{DE}_{15}$ as the responses.

\section{Formulation optimization}

The emulsification time and percentage transmittance measurement were performed as previously described. The dissolution test was performed at $50 \mathrm{rpm}, 37 \pm 0.5^{\circ} \mathrm{C}$, and artificial gastric fluid (AGF) as a dissolution medium. Five hundred milliliters of the SNEDDS filled into the capsule size " 0 " were introduced to the medium. Two milliliters of the sample were taken at intervals of 5, 10, 15, 20, 40, and $60 \mathrm{~min}$. Each sample was replaced with the same amount and temperature of the AGF medium. The sample was filtered through a $45 \mu \mathrm{m}$ membrane filter. One milliliter of SNEDDS was added with $2 \mathrm{ml}$ methanol, homogenized, and the absorbance was measured using a UV-visible spectrophotometer at a wavelength of $281 \mathrm{~nm}$.

The model and effects of the various combinations on the responses were predicted by the equation and contour plots. The optimum formulation was determined by desirability value. The predictive responses of the optimum formulation were verified by comparing them with the experimental responses. The optimum formulation then characterized for the nanoemulsion droplet size, zeta potential, and dissolution efficiency.

\section{Dissolution testing of the optimum formulation}

The dissolution testing was performed in accordance with the $\mathrm{DE}_{15}$ testing described in the previous part of the formulation optimization. The dissolution profile of the capsule containing the optimum formulation was obtained with an average release of PGV-0 concentration versus time of three replications for $60 \mathrm{~min}$. The SNEDDS THC dissolution profile was compared to THC unmodified.

\section{RESULTS AND DISCUSSION}

\section{Preliminary studies}

The preliminary studies were carried out to select the SNEDDS components that had a high THC dissolution capacity so that SNEDDS can load a lot of THC. This could to reduce the dose and minimize side effects. The SNEDDS must also be easily nano-emulsified spontaneously when in contact with the gastrointestinal fluid so it could to increased dissolution, absorption, and bioavailability.

The solubility test result is presented in Fig. 1. The THC showed higher solubility in Labrafil compared with oleic acid. Among the surfactants, the mixture of Kolliphor:Labrasol (1:3) showed the highest capacity to dissolve the THC. It also showed a good compatibility without any separation.

\section{Construction of the pseudoternary phase diagram}

The composition area that was predicted to formed nanoemulsion when the SNEDDS mixed with the water can be seen as the grey area in Fig. 2. In the range of $10-20 \%$, Labrafil showed the selfnanoemulsifying properties with all the concentration of the mix surf and PEG400 (10-80\%). While in the range of $20-30 \%$ of Labrafil, the self-nanoemulsifying properties only showed when it mixed with $30-80 \%$ mix surf and 10-50\% PEG400. The high and low levels for the D-optimal design constraints were set as follows 10 and 30\% for oil (Labrafil), 10 and 80\% for both surfactants (Kolliphor:Labrasol 1:3), and cosurfactants (PEG 400).

\section{Experimental design}

D-optimal design was applied to optimize the SNEDDS of THC more efficient and effective than the trial and error technique. With order to quadratic model fit, in this study D-optimal design requires 12 experimental runs to analyze the experimental error and significance of quadratic fit.

\section{Formulation optimization}

The D-optimal design and the responses are presented in Table 1 . Determination of the optimum formula based on transmittance parameters and emulsification time in aquades, AGF, and AIF media.

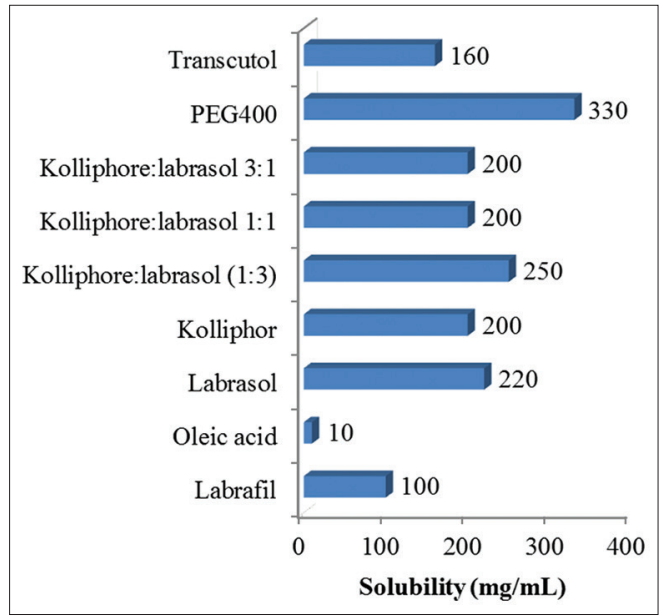

Fig. 1. The tetrahydrocurcumin solubility capacity of various vehicles

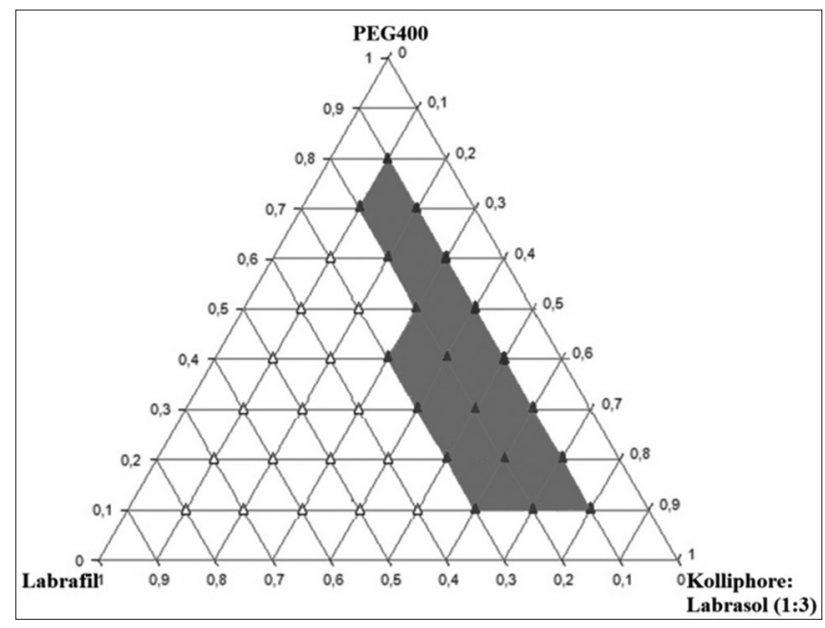

Fig. 2. Pseudoternary phase diagram of Labrafil, Kolliphor: Labrasol (1:3) and PEG400 
Optimization is done using the help of Design-Expert software version 7.1.3 (Stat Ease Inc., Minneapolis). The determination of the optimum formulation is based on the desirability value obtained from the software's calculations. The closer to one the value of desirability indicates that the formula can achieve the optimum formula according to the desired variables.

A successful SNEDDS formulation must be rapidly distributed as nanodroplets in the gastrointestinal fluid. Therefore, the emulsification time determination becomes important [9]

The emulsification time of SNEDDS containing THC is showed in Table 1 . The results showed that all the runs formed nanoemulsion spontaneously with the time required was less than 1 minute. According to Khedekar et al. (2013), the oil-surfactant-cosurfactant system which in less than 2 minutes forms a clear/translucent emulsion is recommended for SNEDDS formulations. If the emulsification time is more than 2 min and produces a cloudy emulsion system, it is not recommended for SNEDDS formulations [9].

The suggested equation for the emulsification time of the SNEDDS was a 2-factor interaction (2FI) (equation 1).

$Y_{1}=1.86 \times 10^{-3}(\mathrm{~A})-1.81 \times 10^{-3}(\mathrm{~B})-4.6 \times 10^{-4}(\mathrm{C})-1.1 \times 10^{-5}(\mathrm{~A})$

$(\mathrm{B})-6.24 \times 10^{-5}(\mathrm{~A})(\mathrm{C})+2.93 \times 10^{-5}(\mathrm{~B})(\mathrm{C})$

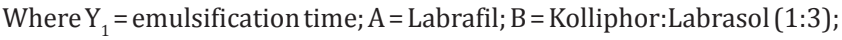
$\mathrm{C}=\mathrm{PEG} 400$

Equation 1 illustrates that increasing the proportion of Labrafil (A) results in increased the emulsification time. The Labrafil had the greatest effect on the emulsification time. Labrafil has hydrophiliclipophilic balance (HLB) of 4; indicate a dominant lipophilic affinity [10], so it is not easily dispersed in water. Otherwise, an increase in mix surf and co-surfactant proportion caused a decrease in the emulsification time. Kolliphor (HLB 13.5) and Labrasol (HLB 14) have high hydrophilicity, lead it to quickly be dispersed in water $[11,12]$. PEG400 is a liquid hydrophilic polymer that often used as cosolvent, so it is also easily dispersed in water [13]. The interaction of Labrafilmix surf and Labrafil-PEG400 reduced the emulsification time. The surfactant molecule decreases the interfacial tension by positioning itself in the oil-water interface so that the oil droplets become more easily dispersed in the water. The more surfactant molecules that interact with oil, the less time it takes for the emulsion to occur. The presence of the PEG400 in oil-water interface increases the fluidity of the interfacial film and facilitates the formation of emulsion [14], while the interaction of mix surf-PEG400 increased the emulsification time. Hydrogen bonds can be formed between surfactants and polyalcohols such as PEG400. Too much interaction between surfactants and cosurfactants causes the interaction energy to decrease due to the self-association mechanism of intermolecular hydrogen bonds, which reduces the ability of $\mathrm{OH}$ alcohol groups to form hydrogen bonds with water. As a result, the dispersed ability will decrease [15].

The influence of factors on the emulsification time is shown in Fig. 3. The increase of Labrafil from $10 \%$ to $30 \%$ decreased the emulsification time from 0.22 to $0.13 \mathrm{~min}$

A good SNEDDS is it forms nanodroplets when dispersed into water. The size of the oil droplet affects the clarity of the emulsion. If the globule size of emulsion system is very small, the light passes through, so the beam of light will be continued so that the solution looks transparent and the resulting transmittance is greater. Aquades do not have particles that resist the transmission of light so that they will pass on the light passing through them without light scattering and have a transmittance value of $100 \%$. The closer to $100 \%$, it is estimated that the emulsion droplets have reached nanometer size [16]

The estimated percentage transmittance for each run composition is calculated by Equation 2 .

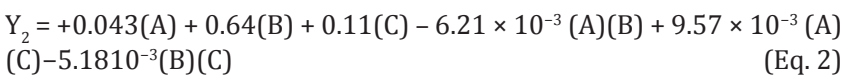

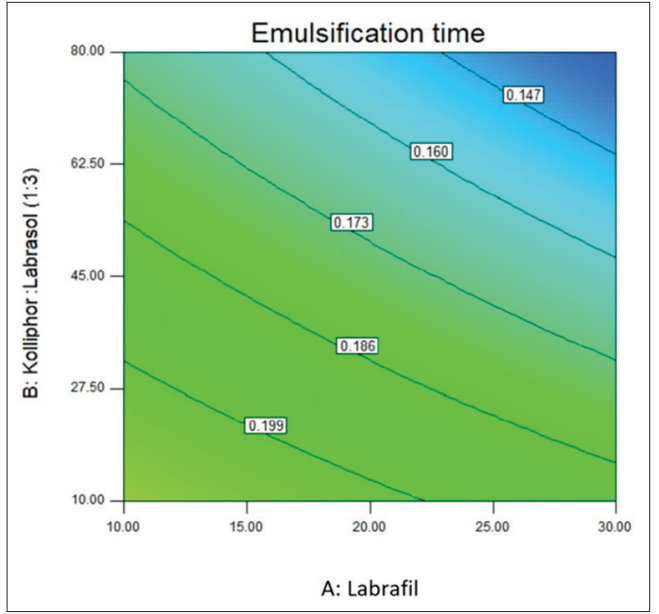

Fig. 3: Effect of self-nanoemulsifying drug delivery system components on emulsification time

Table 1: The actual design of self-nanoemulsifying drug delivery system tetrahydrocurcumin with the response values

\begin{tabular}{|c|c|c|c|c|c|c|c|}
\hline \multirow[t]{4}{*}{ Standard } & \multirow[t]{4}{*}{ Run } & \multicolumn{3}{|c|}{ Factor } & \multicolumn{3}{|l|}{ Response (Y) } \\
\hline & & 1 & 2 & 3 & 1 & 2 & 3 \\
\hline & & $\mathbf{A}$ & B & $\mathbf{C}$ & \multirow[t]{2}{*}{ Emulsification time (min) ${ }^{a}$} & \multirow[t]{2}{*}{$\%$ Transmittance $(\%)^{a}$} & \multirow[t]{2}{*}{$\mathrm{DE}_{15}(\%)^{\mathrm{a}}$} \\
\hline & & $(\%)$ & $(\%)$ & $(\%)$ & & & \\
\hline 12 & 1 & 30 & 10 & 10 & $0.256 \pm 0.02$ & $57.23 \pm 0.03$ & $10.37 \pm 0.02$ \\
\hline 10 & 2 & 10 & 10 & 10 & $0.232 \pm 0.04$ & $52.41 \pm 0.13$ & $10.89 \pm 0.03$ \\
\hline 2 & 4 & 10 & 10 & 10 & $0.245 \pm 0.03$ & $51.43 \pm 0.11$ & $9.41 \pm 0.02$ \\
\hline 11 & 5 & 10 & 10 & 10 & $0.244 \pm 0.03$ & $52.32 \pm 0.10$ & $7.58 \pm 0.02$ \\
\hline 5 & 6 & 30 & 80 & 10 & $0.140 \pm 0.01$ & $80.03 \pm 0.04$ & $42.30 \pm 0.01$ \\
\hline 9 & 7 & 10 & 80 & 80 & $0.220 \pm 0.02$ & $72.63 \pm 0.13$ & $6.91 \pm 0.02$ \\
\hline 4 & 8 & 30 & 10 & 80 & $0.125 \pm 0.02$ & $75.93 \pm 0.24$ & $12.08 \pm 0.03$ \\
\hline 1 & 9 & 10 & 10 & 10 & $0.267 \pm 0.04$ & $53.23 \pm 0.10$ & $9.40 \pm 0.02$ \\
\hline 7 & 10 & 10 & 10 & 80 & $0.177 \pm 0.02$ & $66.54 \pm 0.10$ & $16.85 \pm 0.04$ \\
\hline 6 & 11 & 10 & 80 & 10 & $0.120 \pm 0.01$ & $92.74 \pm 1.33$ & $49.46 \pm 0.12$ \\
\hline
\end{tabular}

${ }^{a}$ mean \pm SD $(n=3)$. DE 1 : Dissolution efficiency at minute 15 
Where $\mathrm{Y}_{2}=\%$ transmittance; $\mathrm{A}=$ Labrafil; $\mathrm{B}=$ mix surf (Kolliphor:Labrasol 1:3); C = PEG400

Equation 2 illustrates the 2FI model. The coefficients of the main effect (A, B, and C) were positive with the highest coefficient of B. This means increasing the concentration of Labrafil, mix surf, or PEG400 resulting in increased percentage transmittance. The mix surf was the most influential component to increasing percentage transmittance. It can be explained by the deposition of surfactant as a monolayer at the oil/water interface above its critical micelle concentration causing a reduction in both the interfacial dilation modulus and the interfacial tension. As a result, the droplet is damaged to smaller and uniform droplets. The beam of light passes through without a lot of scattering, and the transmittance is greater [15].

Visually, the effect of the component ratio on the percentage transmittance is shown in Fig. 4. An increase in the percentage of mix surf from $10 \%$ to $80 \%$ showed non-linear percentage transmittance increase from $58.4 \mathrm{~nm}$ to $82.65 \%$

The last parameter in this optimization is dissolution. It is important for THC in SNEDDS to dissolve both in molecular and micellar form to be ready to absorbed in the gastrointestinal tract. $\mathrm{DE}_{15}$ could be estimated following Equation 3.

$Y_{3}=-0.19(A)+0.58(B)+0.1(C)+1.64 \times 10^{-3}(A)(B)+3.35 \times 10^{-3}(A)$
$(C)-8.08 \times 10^{-3}(B)(C)$

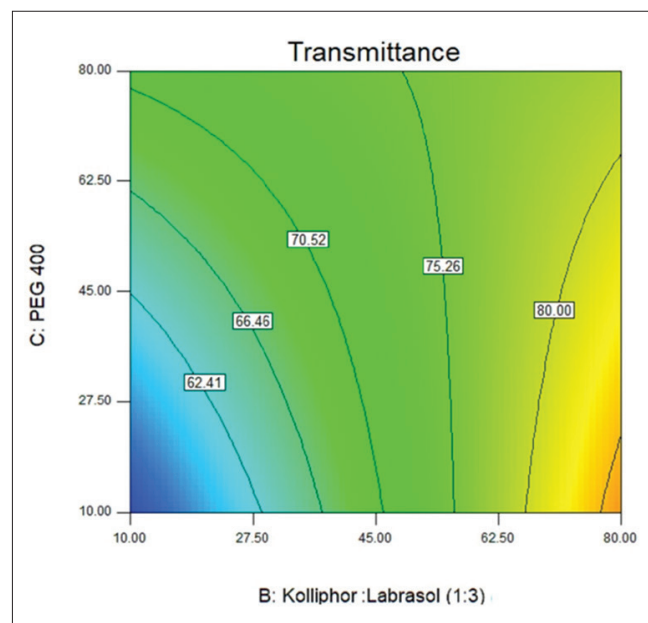

Fig. 4: Effect of self-nanoemulsifying drug delivery system components on percentage transmittance

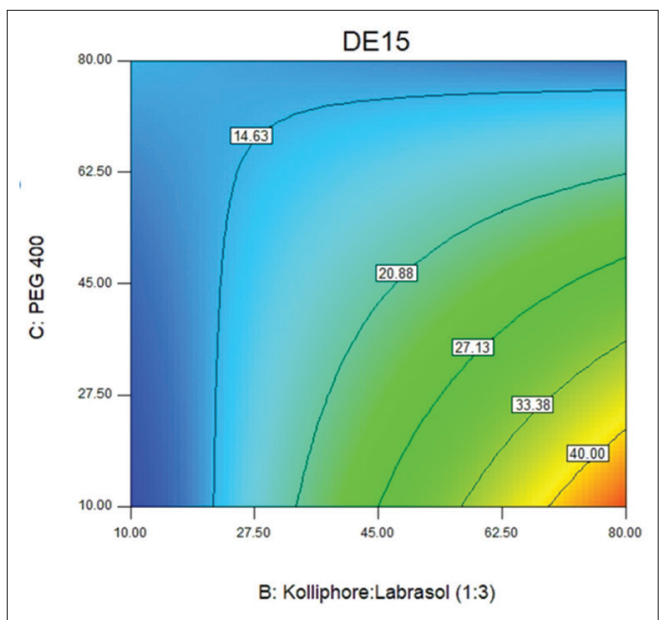

Fig. 5: Effect of self-nanoemulsifying drug delivery system components on dissolution efficiency at minute 15
Where $Y_{3}=D_{15} ; A=$ Labrafil; $B=$ Kolliphor:Labrasol (1:3); C = PEG400.

As seen in Equation 3, the 2FI model applied for the dissolution efficiency of THC at $15 \mathrm{~min}$. Equation 3 contains positive coefficients at main effect $B$ and $C$ and interaction effects of $(A)(B)$ and $(A)(C)$. The positive effect of surfactant and cosurfactant to the dissolution properties is similar to previous reports $[17,18]$.

The effect of SNEDDS component on $\mathrm{DE}_{15}$ is presented in Fig. 5. As seen in Fig. 5 , in the range of $10-80 \%$ mix surf, the $\mathrm{DE}_{15}$ increased from $14.4 \%$ to $45.5 \%$

Overlay plot from the optimization with criteria was emulsification time $<2$ min, percentage transmittance $\geq 80 \%$ and $\mathrm{DE}_{15} \geq 40 \%$, shown in Fig. 6. The yellow area shows the area that meets the criteria.

Simultaneous optimization for all responses is done with using the desirability function. This is calculated by combining individual desirability using geometric averages. A function desirability gives a number between 0 and 1 , with 0 representing the value completely unwanted and 1 represents the ideal response value.

To determine the optimum formulation quantitatively, the desirability function was applied with the criteria of emulsification time to be minimized, percentage transmittance, and $\mathrm{DE}_{15}$ to be maximized. The desirability value is shown in Fig. 7. The maximum desirability was achieved at composition: Labrafil

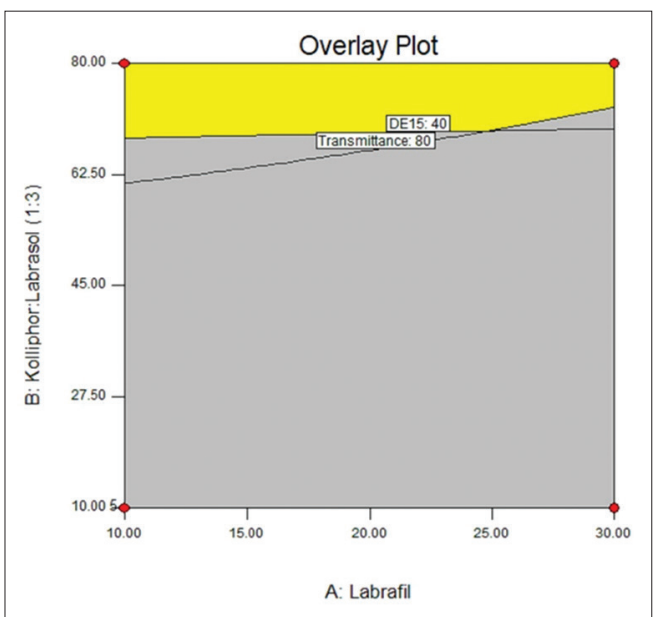

Fig. 6: Overlay plot for the formulation optimization of selfnanoemulsifying drug delivery system containing THC

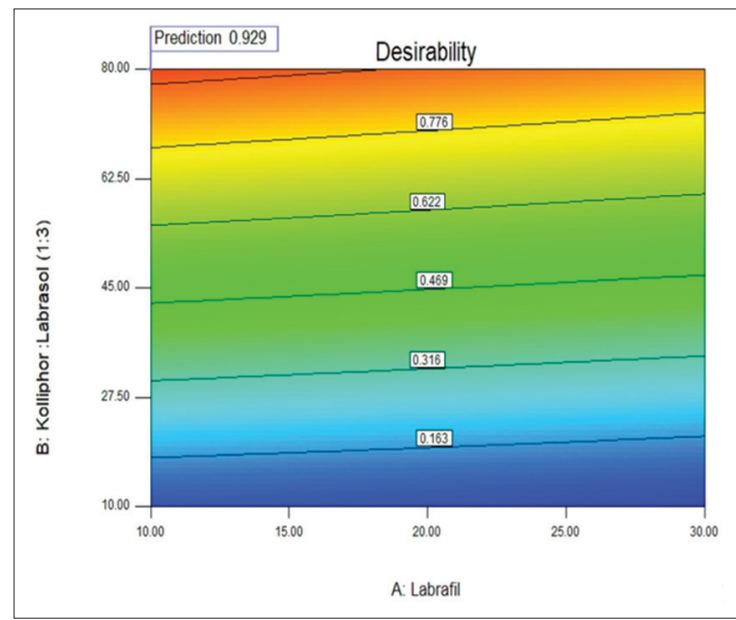

Fig. 7: Desirability value at various compositions 
Table 2: The comparison between the experimental and predictive responses

\begin{tabular}{llll}
\hline Response & Prediction & Experiment & \multirow{2}{*}{ p-value } \\
\cline { 2 - 3 } & & Mean \pm SD & \\
\hline Emulsification time & 0.13 & $0.125 \pm 0.016$ & 0.874 \\
Percentage transmittance & 90 & $91.41 \pm 1.13$ & 0.45 \\
DE $_{15}$ & 46.9 & $49.67 \pm 0.03$ & 0.155 \\
\hline
\end{tabular}

$\mathrm{DE}_{15}$ : Dissolution efficiency at minute 15

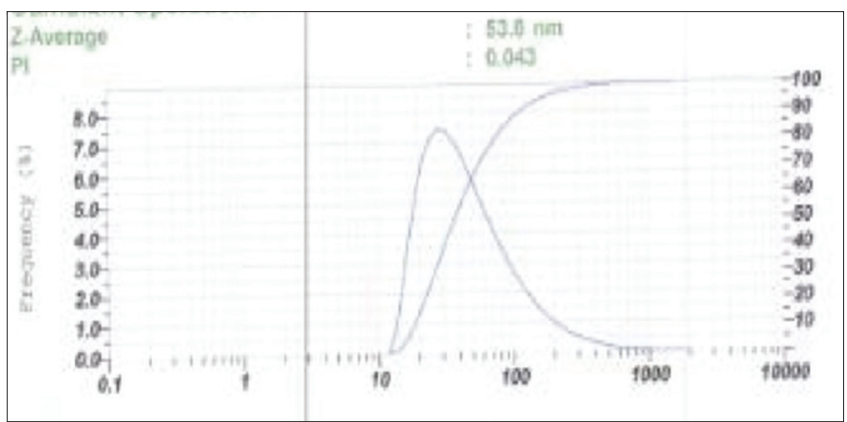

Fig. 8: Results of the droplet size measurements

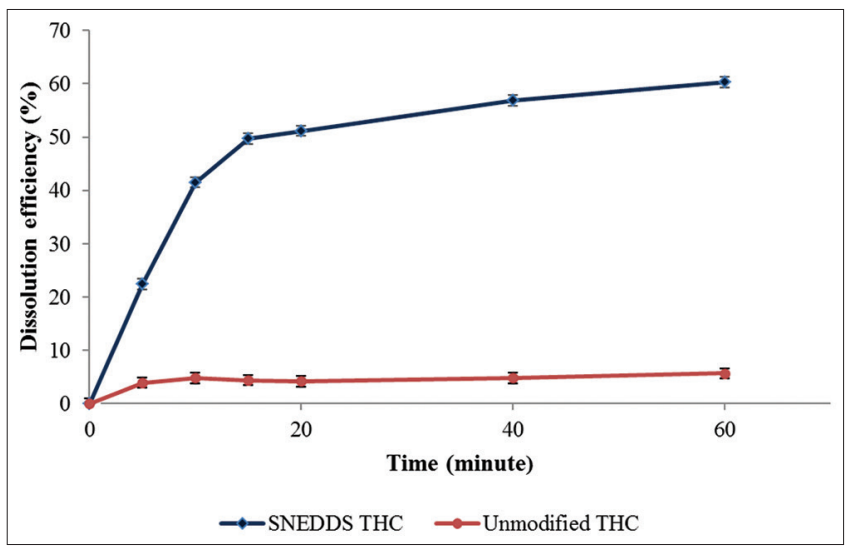

Fig. 9: The dissolution efficiency of self-nanoemulsifying drug delivery system tetrahydrocurcumin (THC) and unmodified THC in AGF medium

10\%, Kolliphor:Labrasol (1:3) $80 \%$ and PEG400 10\% with the corresponding desirability value (D) are 0.929 . The predicted response values of emulsification time, percentage transmittance, and DE15 were $0.13 \mathrm{~min}, 90 \%$, and $49.46 \%$, respectively.

To verify the validity of the model, three batches of the optimum formulation were manufactured, then all responses were assessed. The experimental and predictive responses were compared and analyzed by one-sample t-test and can be seen in Table 2 . All $p>0.05$, so the experimental responses were not significantly different from the predictive responses. The model of each response was valid.

The dropletsize and zeta potential of the optimum formulation of SNEDDS THC are shown in Fig. 8. The droplet of SNEDDS THC had a particle size of $53.8 \mathrm{~nm}$ and met the SNEDDS requirement, i.e. 50-200 nm [19].

The droplet of SNEDDS THC had a particle size of $53.8 \mathrm{~nm}$ and meetS the SNEDDS requirement, i.e. 50-200 $\mathrm{nm}$ [18]. The zeta potential of SNEDDS THC was $-19.4 \mathrm{mVa}$. Normally, a value of $>30 \mathrm{mVa}$ or $<-30 \mathrm{mVa}$ indicate good stability. However, in this study, the stability of SNEDDS THC nanoemulsion was quite good, could be seen in the absence of flocculation. This is because another factor that determines the physical stability is the steric stability that arises due to steric obstacles by a film layer on the surface of the oil droplet.

\section{Dissolution testing of the optimum formulation}

The comparison of the dissolution profile of SNEDDS THC with the unmodified THC is presented in Fig. 9. The optimum formulation of SNEDDS PGV-0 was significantly increased the dissolution $(\mathrm{p}<0.05)$ compared to THC unmodified. This is due to the content of surfactant and cosurfactant in SNEDDS increasing the solubility of THC and stabilizing oil droplets containing THC longer in the liquid bulk. The right composition had also produced nano-sized droplets that have higher solubility.

\section{CONCLUSION}

D-optimal mixture design with desirability function was effective in optimizing the SNEDDS THC. It also generated valid equations and models to describe the effects of the SNEDDS formulation factors on the responses of emulsification time, \% transmittance, and $\mathrm{DE}_{15}$. The SNEDDS formulation significantly enhanced the dissolution of THC solubility.

\section{REFERENCES}

1. Xiang L, Nakamura Y, Lim YM, Yamasaki Y, Kurokawa-Nose Y, Maruyama W, et al. Tetrahydrocurcumin extends life span and inhibits the oxidative stress response by regulating the FOXO forkhead transcription factor. Aging (Albany NY) 2011;3:1098-109.

2. Kumar B, Garg V, Singh A, Pandey NK, Singh S, Panchal S, et al. Investigation and optimization of formulation parameters for selfnanoemulsifying delivery system of two lipophilic and gastrointestinal labile drugs using box-behnken design. Asian J Pharm Clin Res 2018;11:12-8.

3. Caixia W, Jianhua X, Xiaojuan W, Xiuwang H. Tetrahydrocurcumin Solid Dispersion and Preparation Method Thereof. China Patent No. CN101543486A; 2016.

4. Thongyai S, Kaewnopparat N, Songkro S. Influence of polyvinylpyrollidone $\mathrm{K} 30$ on the complexation of tetrahydrocurcumin with hydroxyprolyl $\beta$-cyclodextrin. Naresuan Univ J Sci Technol 2016;24:34-42

5. Xue X, Cao M, Ren L, Qian Y, Chen G. Preparation and optimization of rivaroxaban by self-nanoemulsifying drug delivery system (SNEDDS) for enhanced oral bioavailability and no food effect. AAPS PharmSciTech 2018;19:1847-59.

6. Ganugapati J, Babu R, Ahuja SJ, Mukundan M, Vutukuru SS. Screening and molecular docking studies of curcumin and its derivatives as inhibitors of amyloid-b protein: A key protein in Alzheimer's disease. Asian J Pharm Clin Res 2015;8:98-101.

7. Astuti IY, Marchaban M, Martien R, Nugroho AE. Physical characterization and dissolution study of pentagamavunon-0 loaded self nano-emulsifying drug delivery system. Indones $\mathrm{J}$ Pharm 2018;29:60-5.

8. Montgomery DC. Design and Analysis of Experiments. 8th ed. New York: John Wiley and Sons; 2012.

9. Khedekar K, Mittal S. Self emulsifying drug delivery system: A review. Int J Pharm Sci Res 2013;4:14

10. Mohamed IA, Sultan AS, Hussein IA, Al-Muntasheri GA. Influence of surfactant structure on the stability of water-in-oil emulsions under high-temperature high-salinity conditions. J Chem 2017;2017:1-11.

11. Lo JT, Lee TM, Chen BH. Nonionic microemulsions as solubilizers of hydrophobic drugs: Solubilization of paclitaxel. Materials (Basel) 2016;9:e761

12. Eid AM, El-Enshasy HA, Aziz R, Elmarzugi NA. The preparation and evaluation of self-nanoemulsifying systems containing Swietenia oil and an examination of its anti-inflammatory effects. Int J Nanomedicine 2014;9:4685-95

13. Rowe RC, editor. Handbook of Pharmaceutical Eexcipients. $6^{\text {th }}$ ed. London: APhA, (PhP) Pharmaceutical Press; 2009.

14. Fawzia H, Mona EM, Shaheer M. Ocular drug delivery and the importance of microemulsion as a potential delivery system. Int $\mathrm{J}$ Pharm Chem Sci 2012;1:723-37.

15. Resende KX, Corrêa MA, de Oliveira AG, Scarpa MV. Effect of cosurfactant on the supramolecular structure and physicochemical properties of non-ionic biocompatible microemulsions. Rev Bras Ciênc Farm 2008;44:35-42 
16. Dash RN, Mohammed M, Humaira T, Ramesh D. Design, optimization and evaluation of glipizide solid self-nanoemulsifying drug delivery for enhanced solubility and dissolution. Saudi Pharm J 2015;23:528-40

17. Basalious EB, Shawky N, Badr-Eldin SM. SNEDDS containing bioenhancers for improvement of dissolution and oral absorption of lacidipine. I: Development and optimization. Int J Pharm 2010;391:203-11.
18. Marasini N, Yan YD, Poudel BK, Choi HG, Yong CS, Kim JO, et al. Development and optimization of self-nanoemulsifying drug delivery system with enhanced bioavailability by box-behnken design and desirability function. J Pharm Sci 2012;101:4584-96.

19. Mohd AB, Sanka K, Bandi S, Diwan PV, Shastri N. Solid selfnanoemulsifying drug delivery system (S-SNEDDS) for oral delivery of glimepiride: Development and antidiabetic activity in albino rabbits. Drug Deliv 2015;22:499-508. 